\title{
Embryonal Rhabdomyosarcoma of the Uterine Cervix: Two Cases Report and Literature Review
}

\author{
Khlifi Abdeljalil*, Boukadida Asma, Mouna Kouira, Hachani Faten, Meddeb Sawssen, \\ Hidar Samir, Bibi Mohamed, Essaidi Habib, Khairi Hedi \\ Department of Gynecology and Obstetrics, University Teaching Hospital Farhat Hached, Sousse, Tunisia \\ Email: ${ }^{*}$ khlifiabj@yahoo.fr
}

Received 30 July 2014; revised 25 August 2014; accepted 20 September 2014

Copyright (C) 2014 by authors and Scientific Research Publishing Inc.

This work is licensed under the Creative Commons Attribution International License (CC BY). http://creativecommons.org/licenses/by/4.0/

(c) (i) Open Access

\begin{abstract}
Introduction: Embryonal rhabdomyosarcoma (RMS) of the uterine cervix is a rare and extremely aggressive malignant entity. However, prognosis seems to be improved with the use of multimodality approach treatment. Cases: We report the cases of 50- and 51-year-old women, presenting with vaginal bleeding and mass. The two patients underwent radical surgery. Histological examination revealed RMS of uterine cervix. Treatment was effective in the first case but despite the chemotherapy, the second patient died two months later. Discussion and Conclusions: The prognosis of the cervical embryonal RMS depends on clinical and histological features. The current treatment protocols are based on trials done on pediatric patients. Studies on embryonal RMS treatment and outcomes are limited in women over forty years. In patients with unfavorable prognosis characteristics, the multimodality approach including surgery, adjuvant chemo and radiotherapy can be effective. Otherwise, surgery alone can effectively be proposed.
\end{abstract}

\section{Keywords}

Embryonal Rhabdomyosarcoma, Uterine Cervix, Radiation Therapy, Chemotherapy, Surgery

\section{Introduction}

The rhabdomyosarcoma (RMS) also known as the sarcoma botryoides is a highly malignant tumor arising from embryonal mesenchymea that typically presents as a "grape-like" tumor. It is the most common soft tissue sarcoma in childhood and young adults and accounts for $4 \%$ to $6 \%$ of all malignancies in this age group [1] [2]. It

${ }^{*}$ Corresponding author.

How to cite this paper: Abdeljalil, K., Asma, B., Kouira, M., Faten, H., Sawssen, M., Samir, H., Mohamed, B., Habib, E. and Hedi, K. (2014) Embryonal Rhabdomyosarcoma of the Uterine Cervix: Two Cases Report and Literature Review. Open Journal of Obstetrics and Gynecology, 4, 868-873. http://dx.doi.org/10.4236/ojog.2014.414122 
rarely occurs in the cervix or the uterus and is usually seen in female infants and young children [3]. Few cases of embryonal RMS in women older than 40 years have been described [4]-[8]. We report two cases diagnosed at our center, in perimenopausal women, with different evolution: successful surgery in the first case and a poor prognosis despite the chemotherapy in the second one.

\section{Case Reports}

\subsection{Case One}

A multiparous 50-year-old woman multigravida, presented with irregular vaginal bleeding and pelvic pain since four months. She had neither specific medical history nor chronic illness. The gynecologic examination, under general anesthesia, revealed an 8 to $10 \mathrm{~cm}$ soft, irregular, cauliflower-like fragile mass extending from the posterior lip of cervix, protruding through the vagina. The uterus was increased in size (at half way of the umbilicus) and the parameters were cemented. Pelvic ultrasonography showed normal uterus and adnexa; it concluded to a heterogenic mass measuring $8 \mathrm{~cm}$ in the cervix. An abdomino-pelvic magnetic resonance imaging (MRI) revealed a huge tumor developing in the endocervix, blocking the uterine cavity and invading the myometer and the left parameter. The tumor protruded in the superior third of the vagina (Figure 1(A) \& Figure 1(B)). The histological examination of the biopsy of the lesion was consistent with an embryonal heterologous rhabdomyosarcoma. An immuno-histo-chemical examination showed an intense tumoral cells marking with anti-desmin and anti-myoglobin antibodies. Systemic investigation, including abdominal ultrasonography and chest radiography showed no abnormality. The embryonal RMS was classified in Group III according to the IRS clinical grouping classification (Table 1). The tumor was considered non removable. The patient was subsequently treated with chemotherapy according to the Intergroup Rhadbomyosarcoma Study (IRS) protocols [9]. After
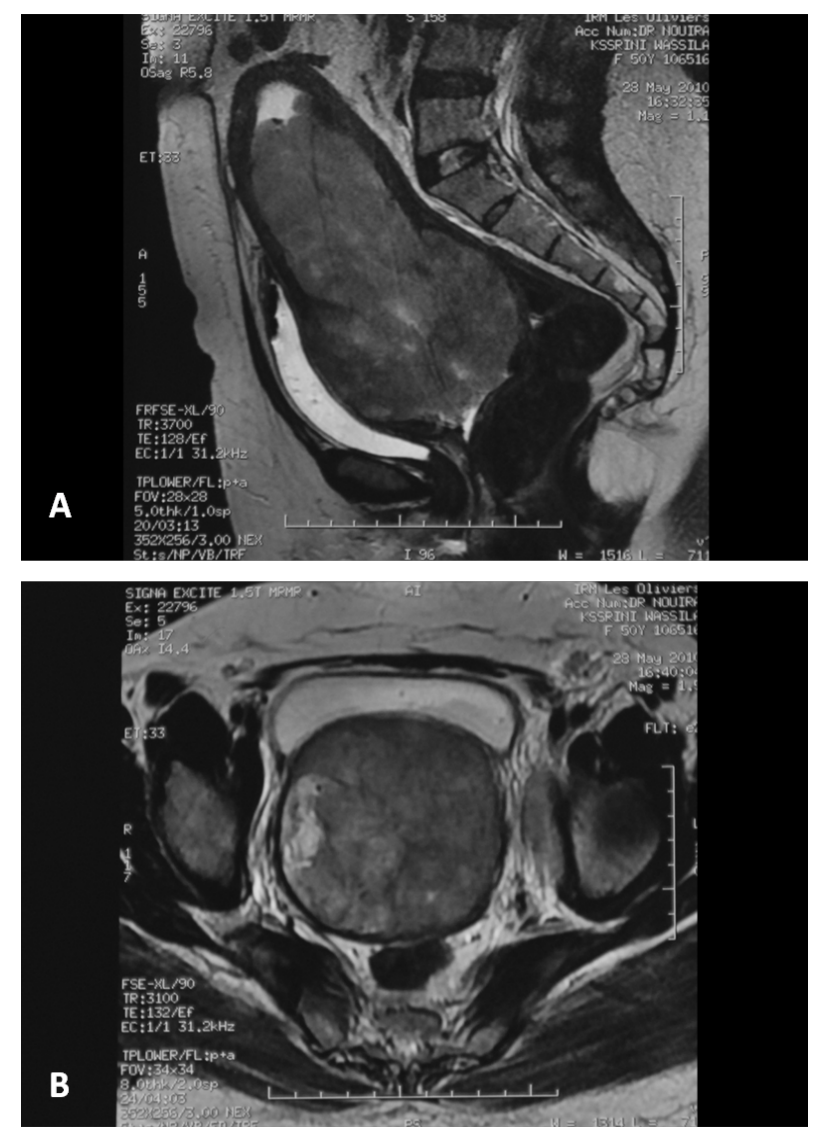

Figure 1. Abdomino-pelvic magnetic resonance imaging (MRI) showing the tumor protruded in the superior third of the vagina. 
Group I: Localised disease, completely resected:

A. Confined to organ or muscle of origin;

B. Infiltration outside organ or muscle origin; regional nodes not involved;

Group II: Compromised or original resections of three types including:

A. Grossly resected tumours with microscopic residual;

B. Regional disease, completely resected, in which nodes may be involved and/or extension of tumour into an adjacent organ present;

C. Regional disease with involved nodes, grossly resected, but with evidence of microscopic residual;

Group III: Incomplete resection or biopsy with gross residual disease;

Group IV: Distant metastasis, present at onset.

four courses of chemotherapy based on Adriamycine and Haloxan, the patient was evaluated. Clinical examination and the pelvic MRI concluded to an extension of the tumor to the uterine corpus and to the bladder with a lymphatic invasion (Figure 2). The patient died two months after the diagnosis.

\subsection{Case Two}

A multiparous, premenopausal, 51-year-old woman presented with vaginal bulk. The gynecologic examination, under general anesthesia, revealed a $5 \mathrm{~cm}$ irregular and partly necrotic mass extending from the left lateral commissure of the cervix, protruding through the vagina. The uterus had no evidence of abnormalities and the parameters were soft. A pelvic MRI showed a tumor developing in the left postero-lateral side of the cervix, blocking the cervical and vaginal canals with no evidence of breaking of the fibrous cervical ring or of the vaginal wall (Figure 3). The tumor was removed for histological examination and the lesion was consistent with an embryonal rhabdomyosarcoma of the cervix. Systemic investigation, including abdominal ultrasonography and chest radiography showed no abnormality. The embryonal RMS was classified in Group IA according to the IRS clinical grouping classification (Table 1). The tumor was considered removable. The patient was subsequently treated with surgery including an extra-fascial hysterectomy extended to the superior third of the vagina associated with a bilateral adnexectomy. No major incidents were reported and the procedure left no residual tumor. The patient is in remission six months after the surgery.

\section{Discussion}

Embryonal rhabdomyosarcoma of the uterine cervix is a rare presentation of the most common soft-tissue sarcoma in the first decades of life [10]. In fact, rhabdomyosarcoma is the most common malignancy of the lower genital tract in young women. The sarcoma botryoides, a subtype of embryonal RMS, accounts for about $10 \%$ of all RMS cases and arises under the mucosal surface of body orifices such as the vagina, bladder and more rarely the cervix. Embryonal rhabdomyosarcoma of the cervix must be distinguished from other rare entities, including adenosarcoma, malignant mixed Mullerian tumor and low-grade stromal sarcoma, as the former has a better prognosis [10]. Three major histological subtypes were recognized by the International RMS Study Group (IRS): embryonal, alveolar and undifferentiated. The IRS group also emitted a clinical classification, the IRS clinical grouping classification as detailed in Table 1.

Although sarcoma botryoides of the cervix has been, mainly, reported in pediatric age, it tends to occur predominantly in older patients with a pick incidence in the second decade. In fact, young women aged 12 - 26 years are mostly affected [11]. Previous reports that included all primary sites showed a poorer five-year disease specific survival for adults with RMS when compared to the pediatric population $(65 \%$ versus $55 \%$; $\mathrm{P}<0.0001)$ [12]. This difference has been attributed to a higher proportion of adverse prognostic clinical and pathological factors, and to inadequate treatment given to adults with RMS [13]. Our patients are older than most of the cases that have been reported concerning embryonal RMS in women older than 40 [4]-[8]. One has been reported in a HIV positive 45-year-old widow and the onset of the tumor at this late age was thought to the due to the patient's HIV positive status and poor state of well-being. The authors emitted the hypothesis that the HIV status may be a clinical condition aiding and abetting rare childhood tumors to manifest and present at adulthood due to the lowered immune system [14]. Our patients had no evidence of immunodeficiency. 


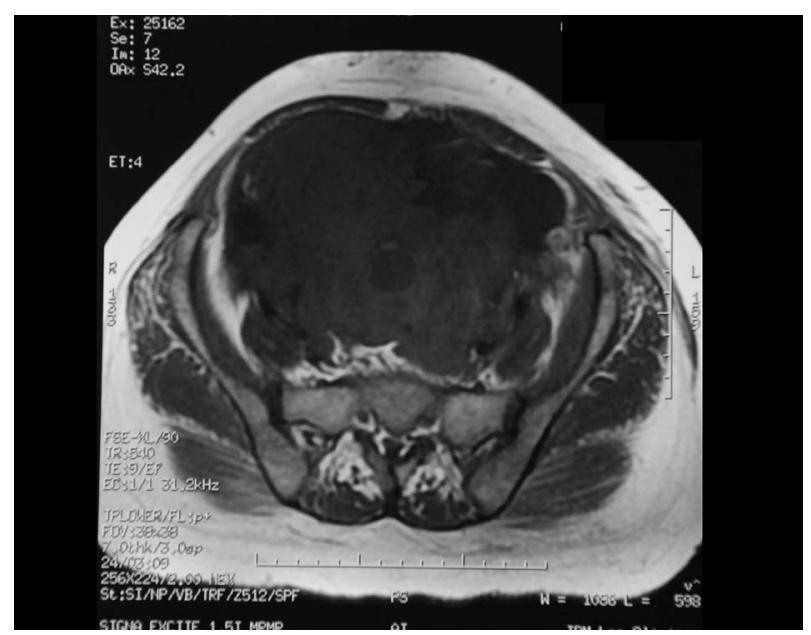

Figure 2. Pelvic MRI showing an extension of the tumor to the uterine corpus and to the bladder with a lymphatic invasion.

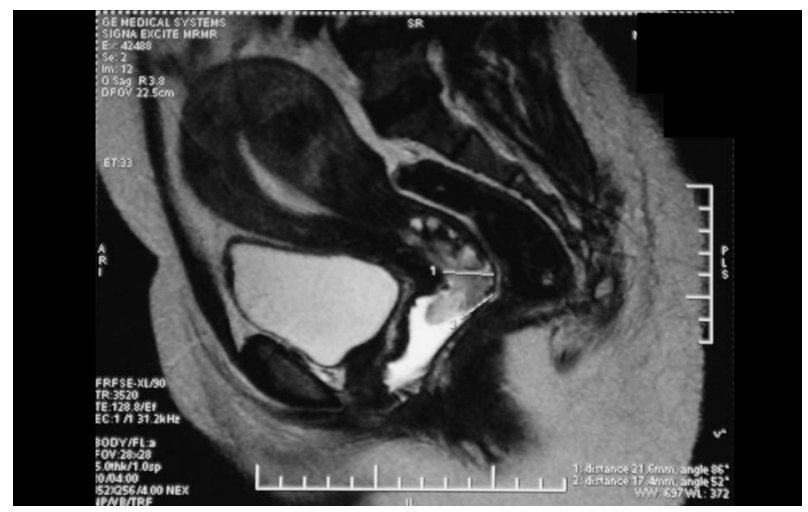

Figure 3. Pelvic MRI showing a tumor developing in the left postero-lateral side of the cervix, blocking the cervical and vaginal canals with no evidence of breaking of the fibrous cervical ring or of the vaginal wall.

Treatment considerations place less concern on preservation of fertility and ovarian function in over 40-yearold patients, but instead have greater concern for the tolerability of treatment with mature bone marrow. The mode of management of cervical RMS has significantly changed over the past 20 years. In 1970, Hilgers et al. [15] recommended that pelvic exenteration as the best treatment for vaginal RMS. The major surgical treatment for RMS which was performed in our first patient consisted of complete resection of the primary tumor with a surrounding "margin" of normal tissue. Wide and complete resection of the primary tumor is less applicable to patients known to have a locally spread and/or metastatic disease at the initial operation, such in our second case. The addition of multiple chemotherapeutic agents as an adjuvant to surgery began in the 1960s. During the following years, authors demonstrated that preoperative chemotherapy with or without radiotherapy can allow preservation of the bladder and rectum, and even save the uterus for fertility with wide local excision of cervix [3]. The most widely used chemotherapy regimen includes vincristine, dactinomycin and cyclophosphamide (VAC). A report by Gordon et al. [3] suggested that between 6 and 12 VAC cycles would allow a reasonable probability for return of menstruation and reproductive function. Although all patients were alive with no evidence of disease 12 to 54 months after primary treatment in the series of Daya et al. [16], the selection criteria for adjuvant chemotherapy were not stated; nor was the indication for primary radical surgery according to Hays et al. [17]. Indeed, there was no consensus for the treatment of the cervical embryonal RMS. The results of recent randomized trials have shown that the introduction of adjuvant chemotherapy in the management of RMS has made a 
substantial contribution to the improvement in survival. But all patients were under age 40 and knowing so, those results might not apply to our case or older patients. We proposed adjuvant chemotherapy to our second patient but, surprisingly, the tumor progressed with a two-month over-all survival. Recently, Adams et al. [18] reported the synchronous association between an embryonal rhabdomyosarcoma of the cervix and a tubular carcinoid tumor of appendicular origin in a 43-year-old woman; adding to the small body of literature surrounding cervical embryonal RMS in women over the age of 40 years and proposing to consider appendectomy during surgical management of the cervical RMS. The optimal management of these tumors is still uncertain owing to their rarity; however, combined modality treatment may result in prolonged survival and cure [19]. The prognosis of cervical SB is more favorable than that of other genital RMS types, particularly when the tumor arises as a single polypoid lesion and the polyp is completely removed. The most important prognostic factors appear to be extent of disease at diagnosis and site of primary tumor [20]. The molecular properties of RMS are being studied for possible prognostic significance. Patients with favorable prognostic factors such as localized disease without deep myometrial invasion, single polyp and embryonal histologic subtype can be treated with minimal invasive surgery although the benefit of adjuvant chemotherapy remains unclear. Patients with unfavorable prognostic parameters seem to benefit from surgery and adjuvant chemotherapy.

\section{Conclusion}

Even if the embryonal RMS of the cervix in peri and post-menopausal women is a rare entity, the diagnosis has to be suspected by both physicians and pathologists in atypical cases. Proper treatment of cervical RMS has not been well assessed, especially in patients over 40 years old, and it is still a subject of debate. Treatment requires a multidisciplinary approach.

\section{Conflict of Interest}

None.

\section{References}

[1] Young, J.L. and Miller, R.W. (1975) Incidence of Malignant Tumors in US Children. Journal of Pediatrics, 86, 254258. http://dx.doi.org/10.1016/S0022-3476(75)80484-7

[2] Maurer, H.M. (1981) The Intergroup Rhabdomyosarcoma Study: Update, Nov. 1978. National Cancer Institute Monograph, 56, 61-68.

[3] Gordon, A.N. and Montag, T.W. (1990) Sarcoma Botryoides of the Cervix: Excision Followed by Adjuvant Chemotherapy for Preservation of Reproductive Function. Gynecologic Oncology, 36, 119-124. http://dx.doi.org/10.1016/0090-8258(90)90121-Z

[4] Miyamoto, T., Shiozawa, T., Nakamura, T., et al. (2004) Sarcoma Botryoides of the Uterine Cervix in a 46-Year-Old Woman: Case Report and Literature Review. International Journal of Gynecological Pathology, 23, 78-82. http://dx.doi.org/10.1097/01.pgp.0000101147.41312.54

[5] Brand, E.B., Nieberg, J.S. and Hacker, R.K. (1987) Rhabdomyosarcoma of the Uterine Cervix. Sarcoma Botryoides. Cancer, 60, 552-560. http://dx.doi.org/10.1002/1097-0142(19871001)60:7<1552::AID-CNCR2820600724>3.0.CO;2-W

[6] Vlahos, N.P.M. and Veridiano, R. (1999) Cervical Sarcoma Botryoides. A Case Report. The Journal of Reproductive Medicine, 44, 306-308.

[7] Ober, W.B. (1971) Sarcoma Botryoides of the Cervix Uteri: A Case Report in a 75-Year-Old Woman. Mount Sinai Journal of Medicine, 38, 363-374.

[8] Sanders, M.A., Gordinier, M., Talwalkar, S.S., et al. (2008) Embryonal Rhabdomyosarcoma of the Uterine Cervix in a 41-Year-Old Woman Treated with Radical Hysterectomy and Adjuvant Chemotherapy. Gynecologic Oncology, 111, 561-563. http://dx.doi.org/10.1016/j.ygyno.2008.07.016

[9] Crist, W.M., Anderson, J.R., Meza, J.L., et al. (2001) Intergroup Rhabdomyosarcoma Study-IV: Results for Patients with Nonmetastatic Disease. Journal of Clinical Oncology, 19, 3091-3102.

[10] Dehner, L.P., Jarzembowski, J.A. and Hill, D.A. (2012) Embryonal Rhabdomyosarcoma of the Uterine Cervix: A Report of 14 Cases and a Discussion of Its Unusual Clinicopathological Associations. Modern Pathology, 25, 602-614. http://dx.doi.org/10.1038/modpathol.2011.185

[11] Smrkolj, S., Rakar, S., Malić, S., et al. (2011) Advanced Embryonal Rhabdomyosarcoma of the Uterine Cervix: A 
Case Report. European Journal of Gynaecological Oncology, 32, 203-205.

[12] Sultan, I., Qaddoumi, I., Yaser, S., et al. (2009) Comparing Adult and Pediatric Rhabdomyosarcoma in the Surveillance, Epidemiology and End Results Program, 1973 to 2005: An Analysis of 2600 Patients. Journal of Clinical Oncology, 27, 3391-3397. http://dx.doi.org/10.1200/JCO.2008.19.7483

[13] Baiocchi, G., Faloppa, C.C., Osório, C.A., et al. (2011) Embryonal Rhabdomyosarcoma of the Uterine Cervix in a 47-Year-Old Woman. Journal of Obstetrics and Gynaecology Research, 37, 940-946. http://dx.doi.org/10.1111/j.1447-0756.2010.01449.x

[14] Anyiam, D.C.D., Ukah, C.O., Onyiaorah, I.V., et al. (2010) Sarcoma Botyroides of the Cervix in a HIV Positive 45Year-Old Woman: A Case Report. Nigerian Journal of Clinical Practice, 13, 341-343.

[15] Hilgers, R.D., Malkasian, G.D. and Soule, E.H. (1970) Embryonal Rhabdomyosarcoma (Botryoid Type) of the Vagina: A Clinicopathologic Review. American Journal of Obstetrics and Gynecology, 107, 484-502.

[16] Daya, D.A. and Scully, R.E. (1988) Sarcoma Botryoides of the Uterine Cervix in Young Women: A Clinicopathological Study of 13 Cases. Gynecologic Oncology, 29, 290-304. http://dx.doi.org/10.1016/0090-8258(88)90228-4

[17] Hays, D.M., Shimada, H., Raney, R.B., et al. (1988) Clinical Staging and Treatment Results in Rhabdomyosarcoma of the Female Genital Tract among Children and Adolescents. Cancer, 9, 1893-1903. http://dx.doi.org/10.1002/1097-0142(19880501)61:9<1893::AID-CNCR2820610929>3.0.CO;2-9

[18] Adams, B.N., Brandt, J.S., Loukeris, K., et al. (2011) Embryonal Rhabdomyosarcoma of the Cervix and Appendiceal Carcinoid Tumor. Obstetrics \& Gynecology, 117, 482-484. http://dx.doi.org/10.1097/AOG.0b013e3182051dd0

[19] Khosla, D., Gupta, R., Srinivasan, R., et al. (2012) Sarcomas of Uterine Cervix: Clinicopathological Features, Treatment and Outcome. International Journal of Gynecological Cancer, 22, 1026-1030. http://dx.doi.org/10.1097/IGC.0b013e31825a97f6

[20] Wieneer, E., Wharam, M., Vietti, T.J., et al. (1995) The Third Intergroup RMS Study. Journal of Clinical Oncology, 13, 610-630. 
Scientific Research Publishing (SCIRP) is one of the largest Open Access journal publishers. It is currently publishing more than 200 open access, online, peer-reviewed journals covering a wide range of academic disciplines. SCIRP serves the worldwide academic communities and contributes to the progress and application of science with its publication.

Other selected journals from SCIRP are listed as below. Submit your manuscript to us via either submit@scirp.org or Online Submission Portal.
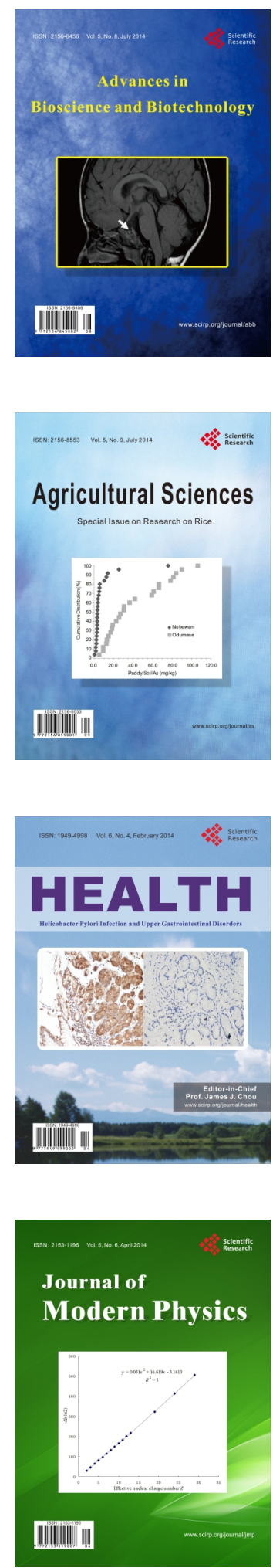
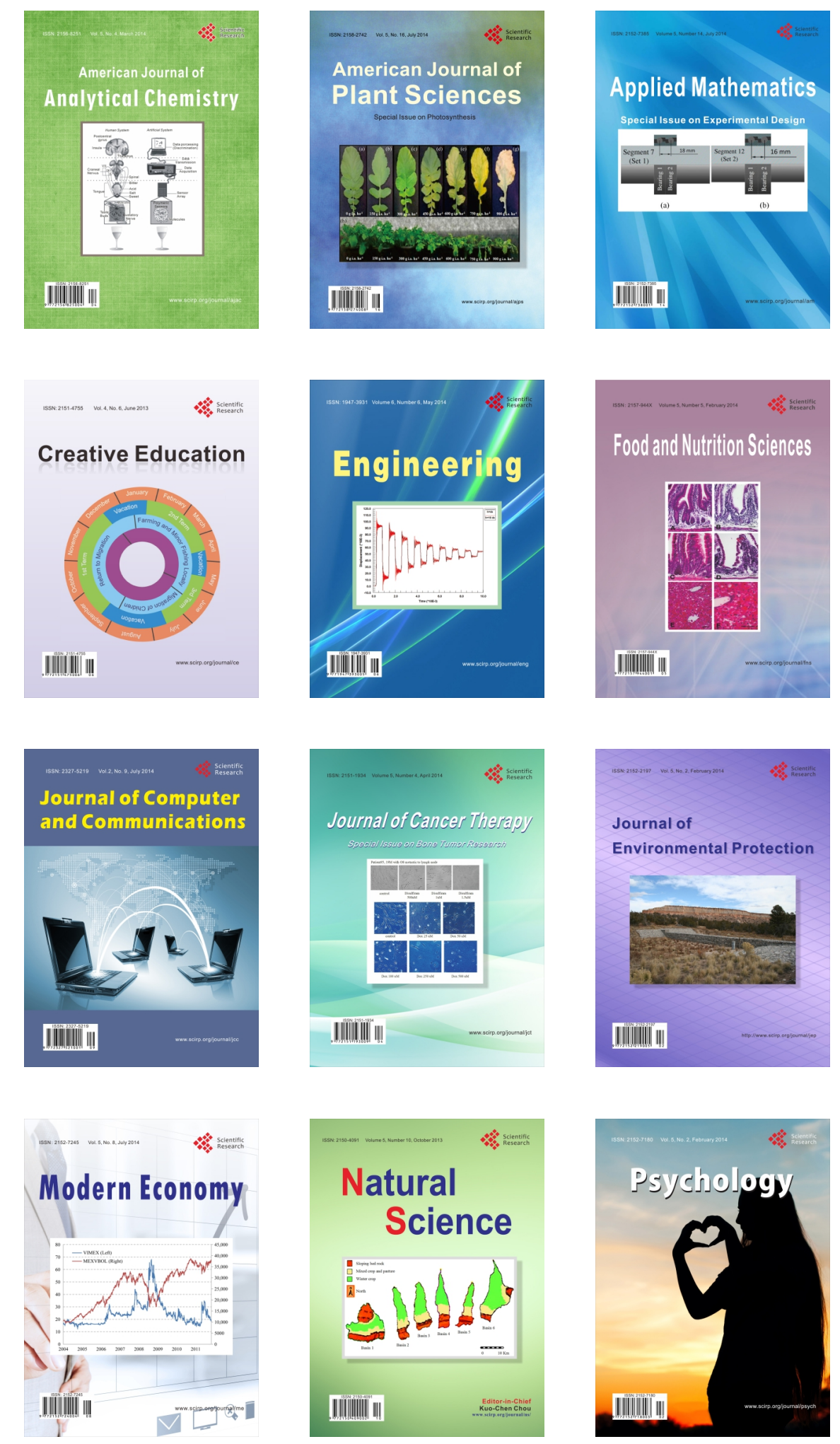